\title{
Tiroidectomía por cáncer: Abordaje trans-disciplinario como factor fundamental para optimizar la calidad de atención en Honduras
}

\author{
Cancer thyroidectomy: Transdisciplinary approach as a key factor in optimizing the quality of care in Honduras
}

\section{Roberto Esquivel-David}

Especialista en Cirugía General, Servicio de Cirugía General, Departamento de Cirugía; Grupo Trans-disciplinario para el manejo de Enfermedades Endocrinas; Hospital Escuela, Tegucigalpa.

\section{INTRODUCCIÓN}

Honduras tiene alta prevalencia de enfermedad tiroidea bocioide, condición identificada desde mediados del siglo pasado $^{1}$ tanto en adultos, como demostrada más recientemente en población infantil. ${ }^{2}$ El Hospital Escuela (HE), Tegucigalpa, es un centro de alto volumen de cirugía tiroidea, con más de 100 procedimientos al año realizados en atención a enfermedades benignas y malignas. En relación a las primeras, las indicaciones quirúrgicas más frecuentes son el hipertiroidismo (Enfermedad de Graves principalmente) y el bocio nodular. El presente artículo se refiere a la experiencia desarrollada por el Grupo Trans-disciplinario para el Manejo de Enfermedades Endocrinas del Hospital Escuela en el manejo de la enfermedad nodular tiroidea, con enfoque particular en el cáncer bien diferenciado de esta glándula en pacientes adultos. Con el propósito de acompañar la conmemoración del Nonagésimo Aniversario de la Revista Médica Hondureña, se preparó este artículo incluyendo una revisión de las publicaciones relacionadas al cáncer tiroideo publicadas en la Revista.

La incidencia de cáncer tiroideo ha tenido un incremento notable desde finales del siglo XX, habiéndose triplicado en Estados Unidos de América (EEUU) entre 1975 a 2016, con una tasa desde $4.8 / 100,000$ habitantes a una de 14.6 por 100,000 habitantes, respectivamente; ${ }^{3}$ para 2019 , en EEUU, $4 \%$ de los nuevos casos de cáncer en las mujeres corresponde al originado en la glándula tiroidea, y es el cáncer más frecuente en mujeres entre 20 y 34 años. ${ }^{4}$ No existen datos recientes en nuestro país; sin embargo, en una serie de 11,840 casos de cáncer en general del periodo 1969-1979, esta malignidad ocupaba el $10 \mathrm{mo}$ lugar en frecuencia, correspondiente al $1.3 \%$ de las neoplasias malignas en mujeres. ${ }^{5}$ En concordancia con la tendencia reportada en la literatura médica extranjera, en nuestro país también ha ocurrido un incremento en el número de

Recibido: 11-02-2020; Aceptado para publicación 05-03-2020

Dirección para correspondencia: Dr. Roberto Esquivel

Correo electrónico: drrobertoesquivel@yahoo.com

Declaración de relaciones y actividades financieras y no financieras y conflictos de interés: ninguna.

DOI: https://doi.org/10.5377/rmh.v88iSupl.1.11674 tiroidectomías realizadas por cáncer tiroideo: en una serie reportada de los años 1982 a 1987 tal diagnóstico ocurrió en una de cada 8 tiroidectomías; ${ }^{6}$ sin embargo, para el año 2017 una de cada tres tiroidectomías realizada en el Hospital Escuela tuvieron diagnóstico preoperatorio de cáncer papilar o sospecha del mismo por biopsia aspirativa con aguja fina (archivo personal del autor).

Debido al excelente pronóstico de sobrevida de esta neoplasia, ${ }^{7,8}$ los aspectos más relevantes del manejo no se refieren a la reducción del riesgo de muerte sino a definir la magnitud de la intervención quirúrgica (resección tiroidea total versus parcial, manejo de la invasión loco-regional), riesgo de complicaciones postquirúrgicas, riesgo de recurrencia y definición de estrategias para el seguimiento., ${ }^{910}$ También debido al buen pronóstico del cáncer papilar tiroideo (el más frecuente), así como a un mejor entendimiento y categorización de los aspectos relacionados a su comportamiento biológico, hemos sido testigos en el último lustro de un de-escalamiento en las recomendaciones de las guías de manejo, ${ }^{10-12}$ planteándose nuevos retos en relación con la efectividad de las estrategias de seguimiento para detección de recurrencia, persistencia o progresión de la enfermedad. ${ }^{13} \mathrm{~A}$ diferencia de otras enfermedades de naturaleza quirúrgica por excelencia que frecuentemente no ameritan manejo coincidente por varias disciplinas (apendicitis aguda, hernias de la pared abdominal, colecistitis calculosa, etc.), la patología quirúrgica tiroidea, antes de ser llevada al quirófano usualmente requiere del filtro del equipo de endocrinología, el cual además se hace cargo de su control metabólico postoperatorio a largo plazo (y seguimiento oncológico en el caso requerido).

\section{CONTEXTO HISTÓRICO LOCAL}

Así como históricamente en el resto de hospitales del país,$^{14}$ desde su fundación no existía en el HE una vía clínica definida para el manejo de los pacientes que eran sometidos a tiroidectomía por cáncer, situación complicada en virtud de la complejidad de la patología en mención, la cual requiere del concurso coincidente de varias disciplinas médicas en diferentes momentos (v.gr. endocrinología, radiología, patología, medicina nuclear y cirugía). De hace algún tiempo ya la literatura reporta mejores resultados si los enfermos son manejados por 
parte de grupos multidisciplinarios, ${ }^{15,16}$ con fluidez de comunicación ${ }^{17}$ y cuando existen vías clínicas definidas para grupos específicos de enfermos, lo que además se traduce en reducción del costo económico al sistema sanitario. ${ }^{18}$ Hasta finales de la primera década del presente siglo, en el HE la interacción entre los diferentes actores (Servicios Clínicos) involucrados se hacía exclusivamente por vía escrita a través del expediente clínico y dependía del cupo asignado por la Sección de Citas, lo cual se traducia en esperas de varios meses para conocer la opinión de los especialistas de los distintos Servicios. Asimismo, la realización de estudios radiológicos y biopsias aspirativas frecuentemente se demoraba varias semanas o meses, y lo mismo ocurría con los informes definitivos de patología, necesarios para proseguir con la toma de decisiones.

Por varios años, en el HE la cirugía tiroidea era realizada, ya sea por cirujanos generales que hacían rotaciones de tres meses cada año por el Sector de Cabeza y Cuello del Servicio de Cirugía General, o como resultado de la captación ocasional de casos por parte de cirujanos generales del área de emergencias. Ninguna de las situaciones mencionadas permitía el desarrollo de las habilidades propias de un cirujano(a) con alto volumen para este procedimiento. Asimismo, debido a que la interrelación con el resto de especialidades ocurría exclusivamente a través de notas escritas en el expediente clínico, aspectos importantes del proceso quirúrgico también eran manejados aisladamente por el cirujano(a), tales como la planificación de la magnitud quirúrgica, los incidentes de la intervención y la descripción de los hallazgos operatorios. Esto abría la posibilidad de que, en el caso de malignidades, los tumores no se estadificaran adecuadamente puesto que algunos detalles indispensables no llegaban a ser del conocimiento del Departamento de Patología ya que la comunicación se hacía exclusivamente a través de la hoja de Solicitud de Biopsia, misma que no contempla ahondar en detalles importantes (existencia de tumor residual macroscópico, invasión a estructuras adyacentes, etc.) aunque los mismos estuviesen debidamente registrados en la Hoja de Descripción Operatoria del Expediente Clínico.

Otro aspecto que tenía manejo sub-óptimo eran las consecuencias endocrinológicas: cuando ocurría hipocalcemia post-tiroidectomía, el Equipo de Endocrinología/Medicina Interna solamente era interconsultado en algunas ocasiones; además, siendo que la mayoría de las intervenciones involucran la resección total de la glándula tiroidea, el manejo del hipotiroidismo postquirúrgico es fundamental, sin embargo, debido a la ausencia de una vía clínica definida, con alguna frecuencia los pacientes eran egresados sin reemplazo hormonal adecuado sino hasta que llegaban al primer control postquirúrgico 0 a la consulta con el Servicio de Endocrinología (lo que ocurriese primero), usualmente no antes de que hubiese transcurrido un mes desde la intervención.

El seguimiento postoperatorio también presentaba escollos ocasionados por la ausencia de una vía clínica sistematizada: los informes finales de patología no eran uniformemente elaborados y además sufrían retrasos, comprensibles si se considera que este Departamento debe responder a la demanda de un hospital que posee más de 1000 camas. También las ci- tas para control postoperatorio por endocrinología eran dadas tardíamente, lo cual se traducía en irregularidades en el protocolo de seguimiento y retrasos para la toma de decisiones sobre la necesidad de recibir yodo radiactivo en los casos que lo requiriesen, así como para el control metabólico. En resumen, la atención brindada a este grupo de pacientes estaba a cargo de diversos actores bien intencionados, pero con falta de sincronía y organizados en "parcelas" aisladas de conocimiento especializado.

\section{EL MODELO TRANS-DISCIPLINARIO}

A partir de 2008, liderados por el Servicio de Endocrinología, y para subsanar las deficiencias del sistema, los médicos involucrados por parte de cada uno de los Servicios Clínicos y de Apoyo construimos canales de comunicación más fluidos que ciertamente tuvieron como efecto una franca agilización en el manejo de los enfermos. En esa época, paulatina e imperceptiblemente la relación del grupo médico se tornó inter-disciplinaria "en tiempo real" (a diferencia de la de tipo multi-disciplinaria, por escrito, y con "prolongados tiempos de espera" que manteníamos antes) caracterizada por una mejor comunicación, revisión de exámenes de laboratorio, imágenes, etc., e intercambio de opiniones mediante mini-reuniones de acuerdo a la necesidad, lo que eventualmente se volvió muy demandante debido al tiempo limitado para cumplir con el resto de la carga laboral que teníamos los médicos involucrados. Es así que, a partir del año 2016, siempre bajo el liderazgo del Servicio de Endocrinología del HE, evolucionamos hacia una relación que consideramos es de tipo trans-disciplinario, ${ }^{19}$ trascendiendo la fragmentación y jerarquización del conocimiento propia de la especialización médica, de tal manera que los diferentes actores, basados en la premisa de que el saber médico debe ser complementario entre las diferentes especialidades para tener una verdadera visión integral y optimizar el manejo de este grupo de enfermos. Además, adquirimos conocimientos pertinentes de las otras especialidades involucradas en este tema (ya no actuando cada uno como poseedor de una parcela aislada del conocimiento especializado en una rama particular de la medicina) y desarrollamos un lenguaje común, generando debates clínicos de alto nivel académico en beneficio de los enfermos, y consecuentemente, de los estudiantes de grado y postgrado, actividad esta que caracteriza los cimientos conceptuales primordiales sobre los que fue creado nuestro HE. ${ }^{20-22}$

\section{CAMBIOS GENERADOS POR EL MODELO TRANS-DISCI- PLINARIO}

Utilizando como fundamento literatura con el mejor nivel de evidencia disponible, el Grupo Trans-disciplinario implementó en el transcurso de los últimos 10 años una serie de componentes en la vía clínica que han tenido impacto significativo en el proceso de atención, entre ellos los siguientes:

1. Definimos el conjunto mínimo de exámenes necesarios en la investigación de los pacientes con nódulos tiroideos, lo que representa una mejora en el uso racional de los medios de diagnóstico. ${ }^{18}$

2. En vista de la relevancia del ultrasonido de alta resolución 
para determinar el riesgo de malignidad de los nódulos tiroideos, adoptamos estándares acordes con la literatura para la categorización ultrasonográfica de nódulos tiroideos de nuestros pacientes. ${ }^{11,23}$

3. A diferencia de lo que se estilaba previamente ${ }^{24} \mathrm{y}$ considerando que la toma de biopsias aspirativas mediante palpación tiene impacto negativo en la sensibilidad del estudio, y en concordancia con la literatura, ${ }^{11,25,26}$ establecimos como norma que las biopsias aspirativas con aguja fina de los nódulos tiroideos y adenopatías sospechosas sean realizadas con guía ultrasonográfica en todos los casos.

4. Diseñamos en consenso una hoja específica para la solicitud de biopsias aspirativas con aguja fina de nódulos tiroideos y adenopatías sospechosas, misma que contiene información útil para que los equipos de radiología y patología optimicen resultados.

5. Adoptamos el Sistema Bethesda para informes de citopatología de nódulos tiroideos. ${ }^{11,27}$

6. Siendo que la hipocalcemia transitoria es la complicación más frecuente de la tiroidectomía, ${ }^{28}$ y debido a que en el HE no siempre hay disponibilidad de reactivos para medición de electrolitos séricos y nivel de parathormona, con fundamento bibliográfico que demuestra su costo-efectividad, ${ }^{29,30}$ durante algún tiempo utilizamos el esquema de profilaxis rutinaria postoperatoria con sales de calcio y vitamina $D$, abordaje que redujo ostensiblemente la ocurrencia de hipocalcemia transitoria postoperatoria. Sin embargo, esto no nos permitía determinar la frecuencia de dicha complicación, por lo que posteriormente decidimos dar manejo individualizado y establecimos como parte de la rutina preoperatoria la identificación y manejo de factores de riesgo para el desarrollo de esta complicación. . $^{30,31}$ Para subsanar la falta de reactivos, hemos tenido el apoyo de la Sociedad Hondureña de Endocrinología, la cual frecuentemente ha realizado donación de exámenes de laboratorio en caso de necesidad (también ha brindado financiamiento para exámenes especiales de laboratorio, tinciones especiales de patología y estudios radiológicos).

7. Establecimos la obligatoriedad del mapeo cervical ultrasonográfico preoperatorio en pacientes con nódulos tiroideos sospechosos/malignos. ${ }^{25}$ Esta herramienta es de suma utilidad para la detección de adenopatías metastásicas, identificación de extensión tumoral local y para identificar anormalidades en la movilidad de las cuerdas vocales, condiciones que pueden modificar el plan quirúrgico.

8. Adicionamos la medición de tiroglobulina en el aspirado de ganglios linfáticos sospechosos para mejorar la sensibilidad del estudio diagnóstico. ${ }^{32}$

9. Tomando en consideración que existe evidencia en la literatura sobre la reducción en la frecuencia de complicaciones cuando la cirugía tiroidea es realizada preferentemente por parte de cirujanos(as) que hagan mayor número de casos al año, ${ }^{33,34}$ más frecuentemente especialistas en cirugía general, cirugía endocrina u otorrinolaringólogos con entrenamiento en cirugía de cabeza y cuello, ${ }^{35}$ deter- minamos que en el HE ésta sea realizada únicamente por cirujanos con alto volumen para dicho procedimiento, en la actualidad dos especialistas en cirugía general y una oncóloga quirúrgica con formación previa en cirugía general.

10. La planificación de la magnitud quirúrgica para cada caso pasó a ser definida mediante consenso de los miembros del grupo trans-disciplinario y fundamentado en las guías de manejo más actualizadas. ${ }^{11}$

11. Los hallazgos operatorios, así como los detalles del procedimiento ahora son comunicados al resto de los miembros del grupo trans-disciplinario de manera rigurosa e inmediata, lo que asegura una mejor estadificación de tumores, estratificación del riesgo de recurrencia y manejo de potenciales complicaciones. ${ }^{16}$

12. Implementamos el uso rutinario de lupas quirúrgicas en la técnica de tiroidectomía, lo que de acuerdo con la literatura está asociado a disminución del riesgo de complicaciones. ${ }^{36}$

13. La técnica tradicional de tiroidectomía mediante uso de gran número de hilos de sutura para ligadura de vasos sanguíneos aumenta el costo y potencialmente el riesgo de infección del sitio quirúrgico, por lo que actualmente la mayoría de tiroidectomías en el HE son realizadas mediante técnica "sin ligaduras", obteniendo control vascular exclusivamente mediante energía bipolar y ultrasónica, lo que se asocia a disminución del tiempo quirúrgico sin aumentar el riesgo de complicaciones de acuerdo a la literatura. ${ }^{37,38}$

14. Debido a que hay evidencia que muestra que el empleo de drenos aumenta la morbilidad sin aportar beneficios luego de tiroidectomía, eliminamos su uso rutinario. ${ }^{39,40}$

15. En contraste con la práctica previa de aplicar profilaxis antibiótica en todos los casos, y tomando en consideración que el riesgo de infección del sitio quirúrgico en este tipo de cirugías no lo justifica, ${ }^{41}$ actualmente solo la utilizamos en los casos que tienen factores de riesgo, representados por una minoría, lo que reduce costos directos al sistema sanitario y potencialmente disminuye el riesgo del desarrollo de resistencia antimicrobiana ocasionada por el uso indiscriminado de tales medicamentos.

16. Con el fin de estandarizar la información obtenida a partir del estudio anatomo-patológico de las piezas quirúrgicas producto de tiroidectomía, adoptamos el sistema de la Academia Americana de Patología para el reporte de neoplasias tiroideas. ${ }^{42}$

17. Basados en la experiencia del Grupo, y en concordancia con guías existentes, ${ }^{30,43}$ instauramos nuestra vía clínica para manejo de hipocalcemia postoperatoria.

18. Como herramienta indispensable para definir las estrategias de manejo inicial y de seguimiento a largo plazo, adoptamos el Protocolo de Estratificación del Riesgo de Recurrencia Tumoral de la guía ATA 2015, cuyo fundamento es la evidencia de que dicho riesgo depende de la presencia de diversas variables identificables una vez se dispone de los hallazgos quirúrgicos y del reporte definitivo de anatomía patológica. ${ }^{11}$ Asimismo, conociendo que la aparición de 
recurrencia / persistencia tumoral también depende de la respuesta al tratamiento inicial, la cual se ve modificada individualmente a lo largo del tiempo, durante el seguimiento utilizamos la Estratificación Dinámica del Riesgo de Recurrencia, ${ }^{44}$ la cual utiliza diversas variables clínicas que se evalúan en distintos momentos de la evolución para tener datos de probabilidad estadística de dicho riesgo.

En el transcurso de los últimos años la relación entre los miembros del Grupo Trans-disciplinario se ha fortalecido mediante reuniones semanales, sistematizadas, con la participación de los médicos especialistas en radiología, patología, endocrinología, medicina interna y cirugía, así como estudiantes de los postgrados respectivos. La dinámica de dichas reuniones inicia con una presentación formal de los casos candidatos a cirugía (por parte de estudiantes del Postgrado de Medicina Interna) que incluyen datos de historia clínica, resultados de exámenes de laboratorio, informe de exámenes radiológicos y de patología, seguido esto del análisis respectivo enriquecido por la participación de todos los miembros del grupo médico. Al final se elabora un plan de acción priorizando cada caso de acuerdo a su naturaleza y a la disponibilidad de cupo quirúrgico. Dicho plan de acción incluye detalles que antes solamente eran manejados por el(la) cirujano, tales como la magnitud de la operación planificada (tiroidectomía total, casi-total, hemi-tiroidectomía), estrategias para reducción de riesgo de hipo-calcemia/ hipoparatiroidismo y de lesión de nervios laríngeos, así como los criterios para el manejo de la invasión locorregional de acuerdo con el mapeo pre-quirúrgico ultrasonográfico.

El desarrollo de la dinámica anteriormente expuesta ha tenido efecto dramático en la reducción de "tiempos" para atención de pacientes, de tal manera que, ya para el año 2017 y hasta finales del 2019, en términos generales el periplo de un(a) paciente desde que llegaba a la consulta externa especializada (endocrinología, cirugía) hasta que tenía plan quirúrgico tomaba aproximadamente una quinta parte del tiempo del que requería en el pasado, excepto en los casos que ameritan estabilización o control de condiciones asociadas (hipo o hipertiroidismo, deficiencia de vitamina $D$, etc.).

Adicionalmente, la creación del espacio trans-disciplinario de intercambio clínico generó el interés por parte del Grupo por compartir nuestra experiencia de atención, de tal manera que el modelo trans-disciplinario pudiese ser aplicado por otros grupos médicos en diferentes latitudes del país en un intento por estandarizar y optimizar el manejo de los pacientes con enfermedad nodular tiroidea. Es así que a la fecha hemos participado en varios eventos académicos en diversas locaciones exponiendo la experiencia adquirida en el curso de los últimos años en relación al abordaje de dicha patología.

El desafortunado desarrollo de la pandemia de COVID-19 ha condicionado una pausa en la eficiente vía de atención que habíamos logrado para este grupo de pacientes, ocasionando un cese completo en la realización de Tiroidectomías en el HE durante los últimos 7 meses, situación similar en otros países, ${ }^{45}$ producto de la preocupación por la seguridad, tanto de pacien- tes (existe evidencia de evolución postoperatoria desfavorable en infectados asintomáticos intervenidos quirúrgicamente ${ }^{46}$ ) como del personal hospitalario. Hasta inicios de octubre 2020 nuestro Grupo ha contabilizado más de 80 pacientes pendientes de cirugía (fuente: registro del Servicio de Endocrinología $\mathrm{HE})$. Indudablemente tal conducta tendrá consecuencias en el pronóstico de algunas de las personas que cursan con cáncer tiroideo puesto que, aunque es una enfermedad característicamente indolente en la mayoría de casos, la ventana de tiempo para su atención no debería extenderse por periodos excesivamente prolongados una vez que el Equipo Trans-disciplinario ha decidido que el caso tiene indicación quirúrgica. Aunque la telemedicina es una propuesta congruente ${ }^{47}$ otro aspecto a considerar son las dificultades que supone su implementación en la vía clínica de atención de estos pacientes, ya que incluye momentos que no pueden realizarse por vía remota: evaluación física preoperatoria, realización de ultrasonido, tomas de biopsia aspirativa y evaluación postoperatoria temprana. Asimismo, debido al contexto económico-social, nuestros pacientes que ameriten radio-yodo aún deben someterse a un periodo de hipotiroidismo de 3-4 semanas pre-aplicación, lo cual ocasiona disfunción inmunológica que los vuelve vulnerables en caso de infecciones virales, además de los efectos propios de la radiación, aunque sobre este último aspecto aún no hay evidencia. ${ }^{48}$

Para el Grupo Trans-disciplinario la situación actual impone nuevos retos para mantener la calidad de atención a las personas que sufren de cáncer tiroideo. Tal como han reportado grupos de otros entornos ${ }^{48-50}$ la implementación de herramientas para estratificación de riesgo de recurrencia en cada caso son fundamentales para lograr este objetivo puesto nos permitirá diseñar estrategias para administrar la crisis de manera óptima, tales como la implementación de vigilancia activa en casos seleccionados, determinación del tiempo permisible de espera para cirugía, la prolongación del tiempo entre estudios ultrasonográficos para seguimiento de nódulos, la necesidad de aplicar yodo radiactivo, entre otros.

\section{CONCLUSIÓN}

El manejo de los pacientes con cáncer bien diferenciado de tiroides requiere del concurso de varias disciplinas que interactúan en momentos puntuales de la línea de tiempo proporcionando información (de laboratorio, radiológica, histopatológica, quirúrgica, médica nuclear) que es integrada por el equipo de endocrinología para determinar el riesgo de recurrencia, tomar decisiones de manejo y definir estrategias de seguimiento. La calidad de esta información está en proporción directa al logro de buenos resultados, así como también lo está la calidad de atención médica brindada.

Inicialmente, la creación del equipo multidisciplinario del HE para el abordaje de patología tiroidea estableció bases para una mejora en la atención. Sin embargo, la migración del equipo hacia el concepto de trans-disciplinariedad ha significado un paso evolutivo de mayor trascendencia para la optimización de recursos y obtención de mejores resultados en los enfermos 
que ameritan cirugía por patología tiroidea, particularmente los que padecen de cáncer en esta glándula. Es importante mencionar que el modelo trans-disciplinario en el manejo de enfermedades complejas también favorece de manera importante las iniciativas para la generación de conocimiento científico de alta calidad. Es muy pertinente el apoyo a la iniciativa de implementar este modelo en otros centros hospitalarios de nuestro país y para el manejo de otras enfermedades en las que se involucran diversas especialidades.

\section{AGRADECIMIENTO}

El desarrollo del esfuerzo colaborativo expuesto en el presente artículo representa la dedicación y tenacidad del siguiente grupo de médicos del HE en el periodo comprendido desde 2008 hasta la actualidad, conformados en el Grupo Trans-disciplinario para el Manejo de Enfermedades Endocrinas, cuyos nombres y departamentos asistenciales se describen a continuación. Servicio de Endocrinología, Departamento de Medicina Interna: Dra. María Alejandra Ramos, Dra. Cristy Sierra, Dra. Dalia Ávila, Dr. Gerson Andino, Dra. Irma de Vicente, Dr. Omar Videa, Dr. Miguel Osejo, Dra. Marlen Cruz, Dr. Luis Rodríguez, Dra. Scarleth Rodas; Departamento de Patología: Dra. Mazlova Toledo, Dra. Yeri Jiménez, Dr. Rubén Valladares, Dra. Flor de Azalea Girón; Departamento de Radiología: Dr. Carlos Rivera, Dr. Leonardo Lara; Servicio de Cirugía General, Departamento de Cirugía: Dra. Bessy Mejía, Dra. Ana Raquel Morales, Dr. Roberto Esquivel.

\section{REFERENCIAS}

1. Borjas EA. Informe general sobre bocio endémico en Honduras y recomendaciones para su prevención. Rev Med Hondur. 1955;23(3):957-64.

2. Avila Montes GA, Posas Vásquez J, Acosta ME. Prevalencia de bocio en niños escolares de Lempira, Honduras. Rev Med Hondur. 1999; 67(3):181-87.

3. National Cancer Institute. Cancer stat facts: thyroid cancer [Internet]. USA: $\mathrm{NIH}$; sf [actualizado 1 agosto 2019; consultado 24 de enero 2020]. Disponible en: https://seer.cancer.gov/statfacts/html/thyro.html

4. American Society of Clinical Oncology. Thyroid cancer: statistics [Internet]. Alexandría: ASCO; 2020. [actualizado 25 julio 2019; consultado 23 de enero 2020]. Disponible en: https://www.cancer.net/cancer-types/thyroidcancer/statistics

5. Bueso Ramos CH, Castillo Hernández JC, Alvarado D. Cáncer en Honduras 1969-1979. Rev Med Hondur. 1983;50(3):136-47.

6. Lagos $\mathrm{CH}$, Arias Brito $\mathrm{C}$. Cáncer de la glándula tiroides revisión de 36 casos. Rev Med Hondur. 1988;56(4):250-53.

7. Siegel RL, Miller KD, Jemal A. Cancer statistics, 2019. CA Cancer J Clin. 2019;69(1):7-34

8. Davies L, Welch HG. Thyroid cancer survival in the United States: observational data from 1975 to 2005. Arch Otolaryngol Head Neck Surg. 2010;136(5):440-4

9. Núñez $E$, Munguía L. Abordaje quirúrgico de nódulos tiroideos con lesión folicular de significado indeterminado. Rev Med Hondur. 2015;83(34):158-64.

10. Roman BR, Randolph GW, Kamani D. Conventional thyroidectomy in the treatment of primary thyroid cancer. Endocrinol Metab Clin North Am. 2019; 48(1):125-41.

11. Haugen BR, Alexander EK, Bible KC, Doherty GM, Mandel SJ, Nikiforov YE, et al. 2015 American Thyroid Association Management Gguidelines for adult patients with thyroid nodules and differentiated thyroid cancer. Thyroid. 2016;26(1):1-133

12. Yeh MW. The year in surgical thyroidology. Thyroid. 2020;30(1):20-4 .

13. Haser GC, Tuttle RM, Su HK, Alon EE, Bergman D, Bernet V, et al. Active surveillance for papillary thyroid microcarcinoma: new challenges and opportunities for the health care system. Endocr Pract. 2016;22(5):602-11.

14. Sánchez JA, Duarte F, Mojica R. Estado actual, prevención y manejo del cáncer en Honduras. Rev Med Hondur. 1992;60(4):171-7.

15. Castejón Cruz OA, Hernández Pacheco HL, Núñez Savoff RA, Bush Wood SW. Dilemas éticos en la práctica clínica quirúrgica y gineco obstétrica. Rev Med Hondur. 2018;86(3-4):138-43.

16. Carty SE, Doherty GM, Inabnet WB, Pasieka JL, Randolph GW, Shaha AR, et al. American Thyroid Association statement on the essential elements of interdisciplinary communication of perioperative information for patients undergoing thyroid cancer surgery. Thyroid. 2012;22(4):395-9.

17. Dos Reiss LL, Tuttle RM, Alon E, Bergman DA, Bernet V, Brett EM, et al. What is the gold standard for comprehensive interinstitutional communica- tion of perioperative information por cancer thyroid patients? a comparison of existing electronic health records with the current American Thyroid Association recommendations. Thyroid. 2014;24(10):1466-72.

18. Zolin SJ, Burneikis T, Rudin AV, Shirley RB, Sipertstein A. Analysis of a thyroid nodule care pathway: opportunity to improve compliance and value of care. Surgery. 2019;166(4):691-97.

19. Libreros Piñeros $L$. El proceso salud enfermedad y la transdisciplinariedad. Rev Cubana de Salud Pública. 2012;38(4):622-28.

20. Aguilar Paz E. El Hospital Escuela (un sueño, una realidad). Rev Med Hondur. 2005;73(Supl 2):S45-S50.

21. Bourdeth Tosta JA. Hospitales de Honduras, evolución cronológica. Rev Med Hondur. 1996;64(4):166-72.

22. Bu Figueroa E. El hospital general de la República 115 años después. Rev Med Hondur. 1997;65(1):44-47.

23. Tessler F, Middleton W, Grant $E$. Thyroid imaging reporting and data system (Ti-Rads): a user's guide. Radiology. 2018; 287: 29-36.

24. Murillo Castillo E. Biopsia por aspiración con aguja delgada(BAAD). Rev Med Hondur. 1998;66(1):24-30.

25. Yeh M, Bauer A, Bernet V, Ferris R, Loevner L, Mandel S, et al. American Thyroid Association statement on preoperative imaging for thyroid cancer surgery. Thyroid. 2015; 25(1):1-14.

26. Wong $\mathrm{C}$, Wheeler $\mathrm{M}$. Thyroid nodules: rational management. World J Surg. 2000; 24(8): 934-41.

27. Cibas E, Ali S. The 2017 Bethesda System for reporting thyroid cytopathology. Thyroid. 2017; 27(11):1341-46.

28. Connolly T, Waters $\mathrm{D}$. Monitoring performance in thyroidectomy: cumulative sum analysis of outcomes. Thyroid. 2010; 20(4): 407-412.

29. Roh J, Park J, Park Ch. Prevention of hypocalcemia with routine oral calcium and vitamin $\mathrm{D}$ supplements in patients with differentiated papillary thyroid carcinoma undergoing total thyroidectomy plus central neck dissection. Cancer. 2009; 115(2): 251-58.

30. Orloff L, Wiseman S, Bernet V, Fahey T, Shaha A, Shindo M Et al. American Thyroid Association statement on postoperative hypoparathyroidism: diagnosis, prevention and management in adults. Thyroid. 2018; 28(7):830-41.

31. Edafe O, Antakia R, Laskar N, Uttley L, Balasubramanian SP. Systematic review and meta-analysis of predictors of post-thyroidectomy hypocalcemia. Br J Surg. 2014; 101(4): 307-320.

32. Grani G, Fumarola A. Thyroglobulin in lymph node fine-needle aspiration washout: a systematic review and meta-analysis of diagnostic accuracy. J Clin Endocrinol Metab 2014; 99(6):1970-82.

33. Adam M, Thomas S, Youngwirth L, Hyslop T, Reed S, Scheri R, et al. Is there a minimum number of thyroidectomies a surgeon should perform to optimize patient outcomes? Ann Surg. 2017; 265(2): 402-07.

34. Kandil E, Noureldine S, Abbas A, Tuffano R. The impact of surgical volume on patient outcomes following thyroid surgery. Surgery. 2013;154(6):1346-53. 
35. Schwartz A. Thyroid surgery: who should do it? how should it be done? Thyroid. 2005; 15(3):185-187.

36. Testini M, Nacchiero M, Piccinni G, Portincasa P, Di Venere B, Lissidini G, et al. Total thyroidectomy is improved by loupe magnification. Microsurgery. 2004; 24(1):39-42.

37. Ruggiero R, Gubitosi A, Conzo G, Gili S, Bosco A, Pirozzi R et al. Sutureless thyroidectomy. Int J Surg. 2014; 12(Supl.1): S189-S193.

38. Antakia R, Edafe O, Uttley L, Balasubramanian SP. Effectiveness of preventative and other surgical measures on hypocalcemia following bilateral thyroid surgery: a systematic review and meta-analysis. Thyroid. 2015; 25(1): 95-106.

39. Maroun CA, El Asmar M, Park S-J, El Asmar ML, Zhu G, Gourin CG, et al. Drain placement in thyroidectomy is associated with longer hospital stay without preventing hematoma. Laryngoscope. 2019. doi: 10.1002/ lary.28269.

40. Salem FA, Almquist M, Nordenström E, Dahlberg J, Hessman O, Lundgren $\mathrm{CL}$, et al. A nested case-control study on the risk of surgical site infection after thyroid surgery. World J Surg. 2018; 42(8): 2454-2461.

41. Uruno T, Masaki C, Suzuki A, Ohkuwa K, Shibuya H, Kitawa W, et al. Antimicrobial prophylaxis for the prevention of surgical site infections after thyroid and parathyroid surgery: a prospective randomized trial. World J Surg. 2015; 39(5): 1282-1287.

42. Mete O, Seethala RR, Asa SL, Bullock MJ, Carty SE, Hodak SP, et al. Protocol for the examination of specimens from patients with carcinomas of the thyroid gland [Internet]. Illinois: College of American Pathologists; 2020. [actualizado agosto 2019; consultado 4 de febrero 2020]. Disponible en: http://www.documents.cap.org/protocols/cp-endocrine- thyroid-19-4200.pdf

43. Meltzer CJ, Irish J, Angelos P, Busaidy NL, Davies L, Dwojak S, et al. American Head and Neck Society Endocrine section clinical consensus statement: north american quality statements and evidence-based multidisciplinary workflows algorithms for the evaluation and management of thyroid nodules. Head Neck. 2019; 41(4): 843-856.

44. Tuttle RM, Alzahrani AS. Risk Stratification in differentiated thyroid cancer: from detection to final follow-up. J Clin Endocrinol Metab. 2019; 104(9): 4087-4100.

45. Lombardi CP, D'Amore A, Grani G, Ramundo V, Boscherini M, Gordini L, et al. Endocrine surgery during COVID-19 pandemic: do we need an update of indications in Italy? Endocrine. 2020; 68(3):485-488

46. Lei S, Jiang F, Su W, Chen C, Chen J, Mei W, et al. Clinical characteristics and outcomes of patients undergoing surgeries during the incubation period of COVID-19 infection. EClinicalMedicine. 2020; 5;21:100331.

47. Ohannessian R, Duong TA. Global telemedicine implementation and integration within health systems to fight the COVID-19 Pandemic: A call to action. JMIR Public Health Surveill. 2020; 6(2):e18810.

48. Raghavan D, Tan AR, Story ES, Burgess EF, Musselwhite L, Kim ES, Clark $\mathrm{PE}$. Management changes for patients with endocrine-related cancers in the COVID-19 pandemic. Endocr Relat Cancer. 2020; 27(9):R357-R374.

49. Vrachimis A, lakovou I, Giannoula E, Giovanella L. Endocrinology in the time of COVID-19: Management of thyroid nodules and cancer. Eur J Endocrinol. 2020; 183(1):G41-G48.

50. Shaha AR. Thyroid surgery during COVID-19 pandemic: Principles and philosophies. Head Neck. 2020; 42(6):1322-1324. 